\title{
Combining levodopa and virtual reality-based therapy for rehabilitation of the upper limb after acute stroke: pilot study Part II
}

Geoffrey Sithamparapillai Samuel ${ }^{1}$, MBBS, MRCP, Nicodemus Edrick $\underline{\text { Oey }}^{2}$, MD, PhD, Min $\underline{C h o o}^{2}$, BEng(Hons), MSc,

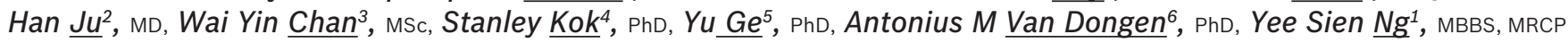

\begin{abstract}
INTRODUCTION This study aimed to evaluate the safety and efficacy of a combination of levodopa and virtual reality (VR)-based therapy for the enhancement of upper limb recovery following acute stroke.

METHODS This was a pilot single-blinded case series of acute stroke patients with upper extremity hemiparesis. Patients were randomised to standard care with concomitant administration of either levodopa alone (control group) or combination therapy consisting of VR-based motivational visuomotor feedback training with levodopa neuromodulation (VR group). Main clinical outcome measures were the Fugl-Meyer Upper Extremity (FM-UE) assessment and Action Research Arm Test (ARAT). Kinematic measurements of affected upper limb movement were evaluated as a secondary measure of improvement.

RESULTS Of 42 patients screened, four patients were enrolled in each of the two groups. Two patients dropped out from the control group during the trial. Patients receiving combination therapy had clinically significant improvements in FM-UE assessment scores of 16.5 points compared to a 3.0-point improvement among control patients. Similarly, ARAT scores of VR group patients improved by 15.3 points compared to a 10.0-point improvement in the control group. Corresponding improvements were noted in kinematic measures, including hand-path ratio, demonstrating that the quality of upper limb movement improved in the VR group.

CONCLUSION Our results suggest that VR-based therapy and pharmacotherapy may be combined for acute stroke rehabilitation. Bedside acquisition of kinematic measurements allows accurate assessment of the quality of limb movement, offering a sensitive clinical tool for quantifying motor recovery during the rehabilitation process after acute stroke.
\end{abstract}

Keywords: dopamine, kinematics, rehabilitation, stroke, virtual-reality

\section{INTRODUCTION}

Stroke is a debilitating condition affecting more than 15 million new patients worldwide annually. ${ }^{(1,2)}$ As one in three affected persons remains permanently impaired due to persistent motor deficits, research is necessary to develop more effective treatments that can increase quality of life for these patients. ${ }^{(3)}$ Early rehabilitation is crucial in maximising functional recovery, ${ }^{(4,5)}$ yet the optimal treatment strategy is still not well defined. ${ }^{(6)}$ Based on the premise that neuroplasticity can be augmented to enhance the rehabilitative process, diverse modalities have been tried as adjunctive treatment for acute stroke, including brain stimulation, robotics, virtual reality (VR) and pharmacotherapy, with varying efficacy. ${ }^{(7,8)}$

Pharmacological modulation, through the use of dopaminergic agents, has been shown to be safe and potentially beneficial in modulating neuroplasticity, although the efficacy of dopaminergic agents as a monotherapy has not been established. ${ }^{(9)}$ On the other hand, VR-based therapy offers high-intensity, repetitive, goaloriented tasks in a stimulating and enjoyable environment. ${ }^{(10)}$ In some trials, VR-based therapy has been shown to be more effective than conventional therapeutic interventions for improving arm function. ${ }^{(11-13)}$ However, there is a paucity of research in the use of VR in the acute period after stroke. Few studies so far have attempted to look at the interaction between pharmacological and VR modalities in a mechanism-driven manner. ${ }^{(10,11)}$

The majority of data from stroke rehabilitation trials has been obtained through the use of clinical scales, such as the FuglMeyer Upper Extremity (FM-UE) assessment; however, notable limitations, such as floor and ceiling effects, have been reported. ${ }^{(14)}$ This is a particular problem in acute stroke rehabilitation, as commonly used scales may not be sensitive enough to detect improvements that occur during a short inpatient rehabilitation stay. ${ }^{(15)}$ Extensive research exploring the clinical relevance of kinematic parameters and their use as outcome measurements has described them as being more sensitive and objective for the assessment of patients' rehabilitation potential. ${ }^{(16,17)}$

We hypothesised that a two-week VR-based intervention for upper limb rehabilitation during the acute post-stroke period would optimise clinical and kinematic outcomes without prolonging the typical duration of post-stroke inpatient rehabilitation. Secondary aims were to: (a) explore the difference between improvements seen in patients receiving VR-based therapy and those undergoing

\footnotetext{
${ }^{1}$ Department of Rehabilitation Medicine, Singapore General Hospital, ${ }^{2}$ Duke-NUS Medical School, ${ }^{3}$ Department of Occupational Therapy, Singapore General Hospital, ${ }^{4}$ Pillar of Information Systems Technology and Design, Singapore University of Technology and Design, ${ }^{5} A^{*}$ STAR Institute for Infocomm Research, ${ }^{6}$ Neuroscience Department, Duke-NUS Medical School, Singapore

Correspondence: Dr Geoffrey Sithamparapillai Samuel, Associate Consultant, Department of Rehabilitation Medicine, Singapore General Hospital, Outram Road, Academia, Level 4, Singapore 169608. Geoffrey.sithamparapillai.samuel@singhealth.com.sg
} 
a comparable amount of conventional occupational therapy; (b) establish the relevance of kinematic measures by investigating their correlation with clinical measures; and (c) assess the acceptance level and overall satisfaction of patients who receive the VR-based rehabilitation modality.

\section{METHODS}

This pilot study was a single-blinded case series of acute stroke patients with upper extremity hemiparesis, who were randomised to standard care and received concomitant administration of either levodopa alone (conventional therapy/ control group) or combination therapy consisting of VR-based motivational visuomotor feedback training coupled with levodopa neuromodulation (combination therapy/VR group). This study compared the tolerability and effectiveness of the combination of VR-based therapy and levodopa against a comparable duration of conventional therapy in patients with hemiparesis due to a recent acute ischaemic stroke within 21 days of symptom onset.

Potential participants were screened from acute stroke patients who were transferred to an inpatient rehabilitation ward. The inclusion criteria were: (a) patients aged 25-99 years, with a first episode of ischaemic stroke within the last 7-21 days; (b) motor power deficit of the affected upper limb assessed using the Medical Research Council scale for muscle strength, with a minimum power of 2; and (c) ability to provide informed consent according to the Mini Mental State Examination, with a score of over 25. Patients were excluded if they were unable to understand study requirements or participate in therapy due to cognitive impairment or aphasia, or had pre-existing motor weakness due to other conditions. Patients with neglect of the affected side were also excluded from the trial. After obtaining informed consent, patients were randomised into the control or VR group using an independent Internet-based random number generator.

All patients received daily standard one-hour sessions of occupational therapy and physiotherapy. In addition, the VR group received 30 minutes of VR-based therapy (with at least 15 minutes of active therapy), whereas the control group received an additional 30 minutes of conventional occupational therapy; each session was conducted daily five days a week for two weeks, as tolerated. The total duration of training in both groups was similar, although we were unable to control the type and intensity of therapy administered during the additional occupational therapy sessions. Intervention sessions were targeted at the affected arm, with patients placed in a sitting position. Both groups were given the trial medication levodopa 1-2 hours before the start of each trial therapy session to allow for adequate absorption and peak plasma levels to be reached by the time the intervention commenced. The pharmacotherapy administered was a single daily dose of $125 \mathrm{mg}$ Madopar (Roche Products Limited, Welwyn Garden City, Hertfordshire, UK), which consisted of a combination of $100 \mathrm{mg}$ levodopa and 25 mg benserazide. ${ }^{(18)}$

VR-based therapy consisted of a specially developed software program that encouraged active elbow flexion and extension

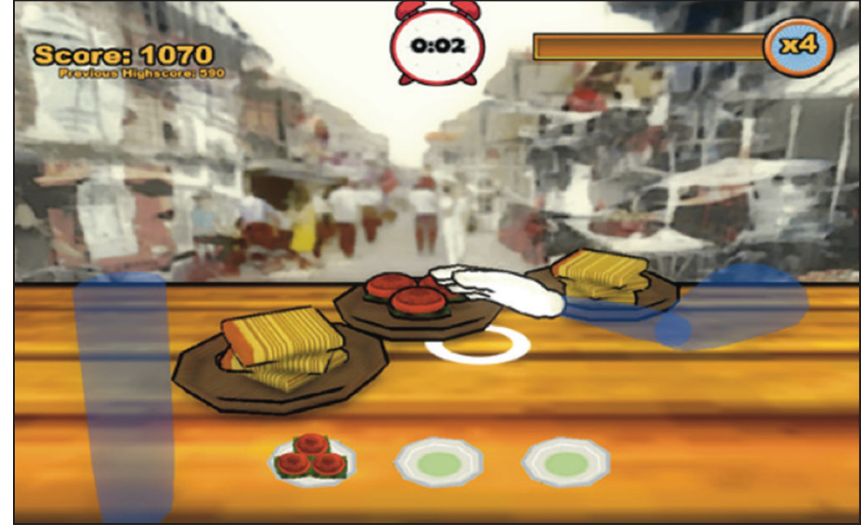

Fig. 1 Screen capture of the virtual reality-based game shows plates of local delicacies and the avatar arm reaching for the food items. The patient's total score for this session, remaining playing time and score multiplier indicator are visible in the upper portion of the screen.

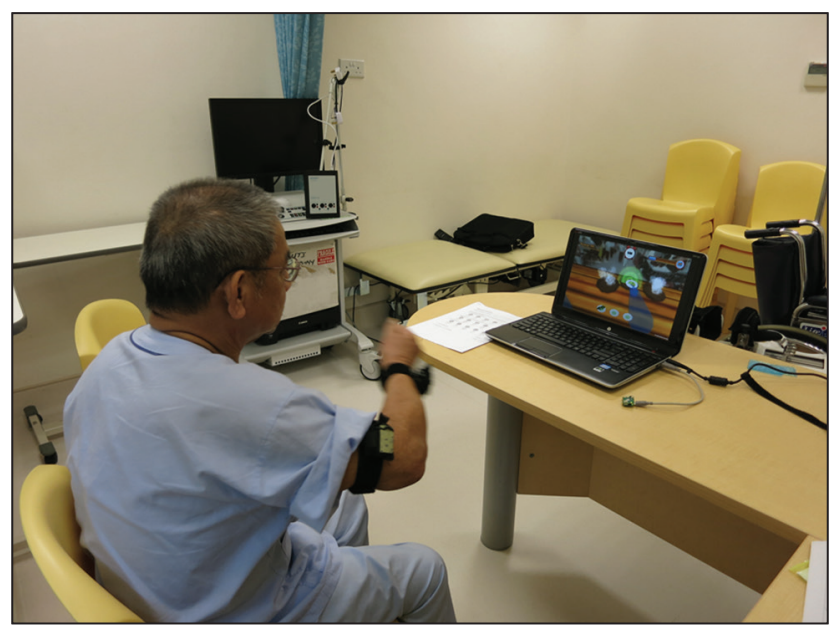

Fig. 2 Photograph shows a patient engaged in the virtual reality-based game with two inertial measurement units strapped to his right arm (above the elbow) and forearm (above the wrist). A wireless receiver can be seen on the table adjacent to the notebook computer.

movements of the affected arm by simulating the arm bringing as many food items to the mouth as possible within a 90-second interval (Fig. 1). The software interface was a custom-developed system coded using the three-dimensional game engine developed by Unity Technologies (San Francisco, CA, USA). This visuomotor interface featured several built-in positive reinforcement signals, in the form of audiovisual feedback each time a successful attempt was made, and encouragement to reach for food items at different locations (to increase the range of motion) through the use of a points system. Progression of therapy was encouraged through the 'unlocking' of new food items as the patient's points reached varying levels and the provision of a score multiplier, which could be achieved by collecting combinations of food items.

Patients interacted with this program via wireless inertial measurement units (IMUs) strapped to their affected arm and wrist (Fig. 2). We utilised IMU devices based on the InvenSense MPU-9150 chip (InvenSense, San Jose, CA, USA), a state-of-the-art integrated nine-axis motion tracking device combining a three-axis MEMS (microelectromechanical systems) gyroscope (InvenSense, San Jose, CA, USA), three-axis MEMS accelerometer (InvenSense, San Jose, CA, USA), three-axis 
MEMS magnetometer (InvenSense, San Jose, CA, USA) and the InvenSense Digital Motion Processor ${ }^{\mathrm{TM}}$ (DMPTM; InvenSense, San Jose, CA, USA) hardware accelerator engine. We selected these IMUs as they were small and easily wearable (each IMU weighed $15 \mathrm{~g}$ ), and for their ability to sample a wide range of data. The device featured a user-programmable gyroscope with a full-scale range of up to $\pm 2,000 \%$ second and an accelerometer with a range of up to $16 \mathrm{~g}$ force. For the purposes of this study, motion data was transmitted wirelessly to the receiver at a $50-\mathrm{Hz}$ sampling rate. Captured kinematic data included angular velocity, acceleration and position in three-dimensional space. The high sensitivity recording was able to capture minute movements of the elbow joint, hence allowing the patient's movements to correspond to the movements of an in-game virtual arm and creating a semi-immersive VR environment in which real-time $x, y$ and $z$ coordinates of the joints were continuously sampled for calculation of kinematic measurements.

Patients in the VR group played a 90-second VR game ten times during each session for a total activity duration of 15 minutes. They were allowed to rest for up to five minutes between sessions. Patients were instructed to rest between games to lessen the possibility of fatigue-induced bias. A study coordinator accompanied patients at all times to ensure patient safety in case of injury or photosensitivity-induced seizures from the virtual environment.

Before each therapy session, calibration was performed to record the range of motion of the patient's arm, to account for individual and per session differences as the patient improved. Calibration also served to ensure that each session impelled the patient to perform at a skill level that was neither too challenging nor effortless, allowing for optimal motor learning. In addition, the virtual arm extended completely when the calibrated range was reached, even if the patient's arm was not doing so. This observation of an ideal action provided augmented feedback and activated the mirror neuron system, which are recognised factors for motor learning. ${ }^{(18)}$

To track the efficacy of VR intervention with the gaming system, clinical and kinematic measurements were obtained at baseline and post intervention for all patients. To assess patients' acceptance of and satisfaction with the new rehabilitation modality, a short questionnaire was also administered for VR group patients before and after intervention. All measurements were taken within a mean duration of $1.8 \pm 0.5$ days from the initiation or conclusion of the intervention course.

Clinical assessments were performed by an occupational therapist blinded to the assigned treatment arm. Two clinical measures were chosen to sensitively monitor improvements in different domains of the World Health Organization International Classification of Functioning, Disability and Health, namely that of body function (i.e. FM-UE assessment) and activity (Action Research Arm Test [ARAT]). These are clinically validated assessment tools for the analysis of stroke recovery. ${ }^{(19)}$ The FM-UE assessment score has a range of 0-66 and assesses reflexes, synergy of movements and coordination of upper limb movements. The ARAT has a maximum score of 57 and measures the ability to perform gross upper limb movements and to grip, grasp and pinch objects, and individual activity scores. For both scales, better ability is indicated by a higher score.

Kinematic assessments were obtained from raw data recorded by the wearable sensors, which consisted of timestamped position data ( $x, y, z$ coordinates) of the elbow and wrist joints. As the IMUs were sampled at a rate of $50 \mathrm{~Hz}$, this translated into a large dataset of 4,500 data points for every 90-second game. To translate this data into meaningful kinematic measurements, the hand trajectories and velocity profiles were plotted out for visual inspection and were crosschecked to ensure accuracy. Kinematic measures calculated were: (a) hand-path ratio (HPR); (b) number of velocity peaks (VPs); (c) time taken to complete each flexion movement ('time taken'); and (d) the total number of flexions performed during each game ('flexions').

HPR is a surrogate marker for movement quality, which is defined as the actual length of the path traversed divided by the shortest distance between the start and end points. ${ }^{(19)} \mathrm{A}$ healthy person should have a HPR close to 1, choosing the most optimal path of a straight line to travel between two points. The same parameter has been used in prior literature under different terms ${ }^{(20)}$ and has been shown to approach a ratio of 1 as stroke patients recover.

Number of VPs is another parameter that has been frequently used in the literature based on the understanding that stroke patients' movements tend to comprise multiple sub-movements or frequent mid-movement correction attempts due to a deficit in descending motor commands and ascending sensory feedback. ${ }^{(16,17,19)}$ Instantaneous velocities were computed, smoothed and transformed into VPs, which were defined as samples for which the two preceding and two succeeding smoothed samples were monotonically rising and falling, respectively. ${ }^{(21,22)}$ The number of VPs present during each flexion movement was then summed up and tabulated.

At the end of the trial, a questionnaire was administered to patients in the VR group, which included questions on previous experience with computers, whether patients felt that VR therapy was beneficial and engaging, and if it should be part of routine therapy. Each question in the questionnaire was presented on a five-point Likert scale.

\section{RESULTS}

From January to May 2014, 42 patients were screened for participation in the trial. However, 34 patients were excluded, as 24 did not meet the inclusion criteria and ten patients refused consent. As such, eight patients were enrolled, with four patients randomly assigned to each treatment group. None of the patients in the VR group complained of any discomfort from interaction with the virtual environment. In the control group, two patients dropped out after Sessions 2 and 8, respectively, for personal reasons not related to the trial. The patient recruitment process is illustrated in Fig. 3.

The demographics of the study population are shown in Table I. The mean age of the patients was 63.3 years, and mean 
Table I. Demographics of the trial patients.

\begin{tabular}{llllllcc}
\hline Group & Patient no. & Gender/age (yr) & $\begin{array}{l}\text { Affected } \\
\text { side }\end{array}$ & $\begin{array}{l}\text { Stroke } \\
\text { location }\end{array}$ & $\begin{array}{l}\text { Clinical stroke } \\
\text { classification }\end{array}$ & $\begin{array}{c}\text { Duration between } \\
\text { stroke \& start of } \\
\text { trial (day) }\end{array}$ & $\begin{array}{c}\text { No. of therapy } \\
\text { sessions completed }\end{array}$ \\
\hline VR & 2 & $\mathrm{~F} / 72$ & $\mathrm{~L}$ & Brainstem & Ataxic hemiparesis & 12 & 10 \\
& 4 & $\mathrm{M} / 63$ & $\mathrm{R}^{*}$ & Subcortical & Ataxic hemiparesis & 6 & 6 \\
& 5 & $\mathrm{M} / 74$ & $\mathrm{~L}$ & Subcortical & Pure motor & 12 & 9 \\
Control & 7 & $\mathrm{M} / 60$ & $\mathrm{R}$ & Brainstem & Sensorimotor & 5 & 10 \\
& 1 & $\mathrm{M} / 63$ & $\mathrm{~L}$ & Subcortical & Ataxic hemiparesis & 7 & 8 \\
& 3 & $\mathrm{M} / 67$ & $\mathrm{R}^{*}$ & Subcortical & Ataxic hemiparesis & 6 & $8^{+}$ \\
& 6 & $\mathrm{M} / 68$ & $\mathrm{~L}^{*}$ & Subcortical & Pure motor & 10 & $2^{+}$ \\
& 8 & $\mathrm{M} / 39$ & $\mathrm{R}$ & Cortical & Pure motor & 10 & 9 \\
\hline
\end{tabular}

*Dominant hand affected. +Dropped out of trial. F: female; L: left-sided weakness; M: male; R: right-sided weakness; VR: virtual reality

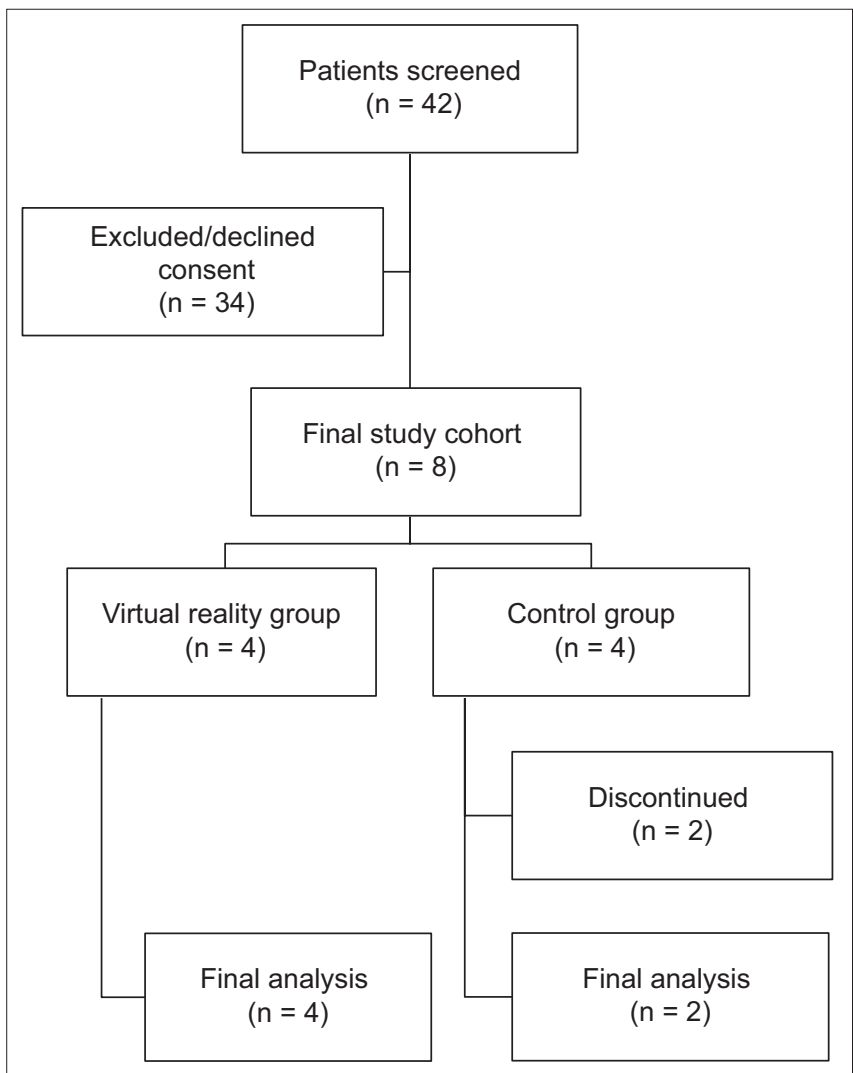

Fig. 3 Flowchart shows the recruitment of patients into the virtual reality and control groups.

time from stroke onset to enrolment was $8.7 \pm 1.3$ days. The mean number of therapy sessions received before discharge was $8.8 \pm$ 0.9 in the VR group and $6.8 \pm 1.0$ in the control group.

VR group patients showed improvement in mean FM-UE score from 36.5 to 53.0 (by 16.5 points), approaching the maximum achievable score of 66. Mean ARAT score for the VR group improved from 15.5 to 30.8 (by 15.3 points). Comparatively, the control group showed improvement in mean FM-UE score from 62.0 to 65.0 (by 3.0 points) and mean ARAT score from 42.5 to 52.5 (by 10.0 points). The FM-UE and ARAT scores for both the control and VR groups are shown in Fig. 4. The minimal clinically important difference for FM-UE and ARAT scores has been established at 4.25-7.25(23) and 12-17 points, respectively, ${ }^{(24)}$ regardless of whether the affected hand is dominant and non-dominant. The 16.5- and 15.3-point increases
Table II. Individual patient differences in motor power before and after intervention.

\begin{tabular}{|c|c|c|c|c|c|c|c|c|}
\hline \multirow[t]{3}{*}{ Motor power } & \multicolumn{8}{|c|}{ Patient no. } \\
\hline & \multicolumn{4}{|c|}{ VR group } & \multicolumn{4}{|c|}{ Control group } \\
\hline & 2 & 4 & 5 & 7 & 1 & 3 & 6 & 8 \\
\hline Pre-trial MMT & 2 & $4+$ & $4+$ & 3 & $4+$ & 3 & 2 & $4+$ \\
\hline Post-trial MMT & $4+$ & 5 & $4+$ & 4 & 5 & $N A^{*}$ & $N A^{*}$ & 5 \\
\hline
\end{tabular}

*Patient dropped out of study. MMT: manual motor power testing; NA: not available; VR: virtual reality

achieved in this trial for the respective FM-UE and ARAT scores of VR group patients were thus clinically significant.

Based on clinical observation, two of the four VR group patients initially had great difficulty performing anti-gravity movements and required anti-gravity support at their elbows, but were progressively able to play entire games without assistance. Their range of motion also increased, from originally only being able to reach the nearest virtual plate to freely traversing across all virtual plates. The results of manual motor power testing, as performed by an independent assessor at the start and end of the trial, are presented in Table II.

Kinematic data showed corresponding improvements that matched the clinical data results (Fig. 5). For the VR group, mean HPR improved from 2.27 to 1.41 (improvement $-0.86 \pm 0.29$ ). The control group had an initial mean HPR of 1.17 and a postintervention mean HPR of 1.18 (change $0.02 \pm 0.06$ ), which was very close to the optimum HPR of 1 and suggested good movement of the affected limb both pre- and post-intervention. For the VR group, there were improvements in mean number of VPs from 2.63 to 1.34 (change $-1.30 \pm 0.23$ ); mean time taken from 0.59 seconds to 0.41 seconds; and mean flexions per 90 -second game from 13.2 to 36.9. For the control group, there were corresponding improvements in mean number of VPs from 1.38 to 1.01 (change $-0.37 \pm 0.70$ ); mean time taken from 0.40 seconds to 0.31 seconds; and mean flexions per 90-second game from 25.3 to 29.5.

Daily observation of patients undergoing VR therapy showed that they enjoyed the sessions and looked forward to them. None of the patients had any prior experience with conventional gaming systems, and some of the patients who were aged $\geq 60$ years were initially apprehensive about using computers. Despite this, feedback from the questionnaire was highly encouraging. Patients 

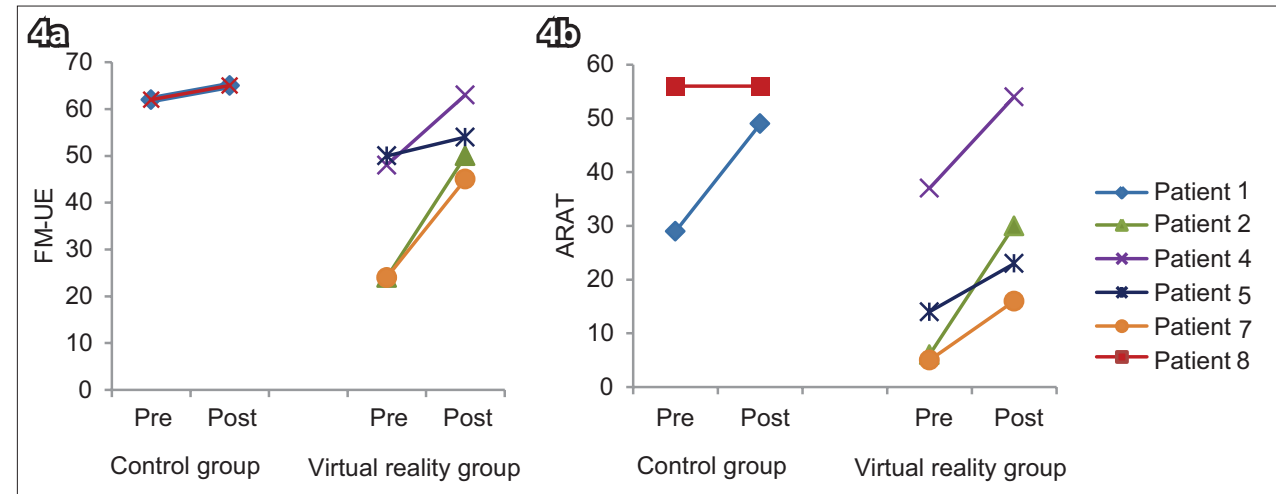

Fig. 4 Plot graphs show the (a) Fugl-Meyer Upper Extremity (FM-UE) assessment and (b) Action Research Arm Test (ARAT) scores achieved by control and virtual reality (VR) group patients pre- and post-rehabilitation.

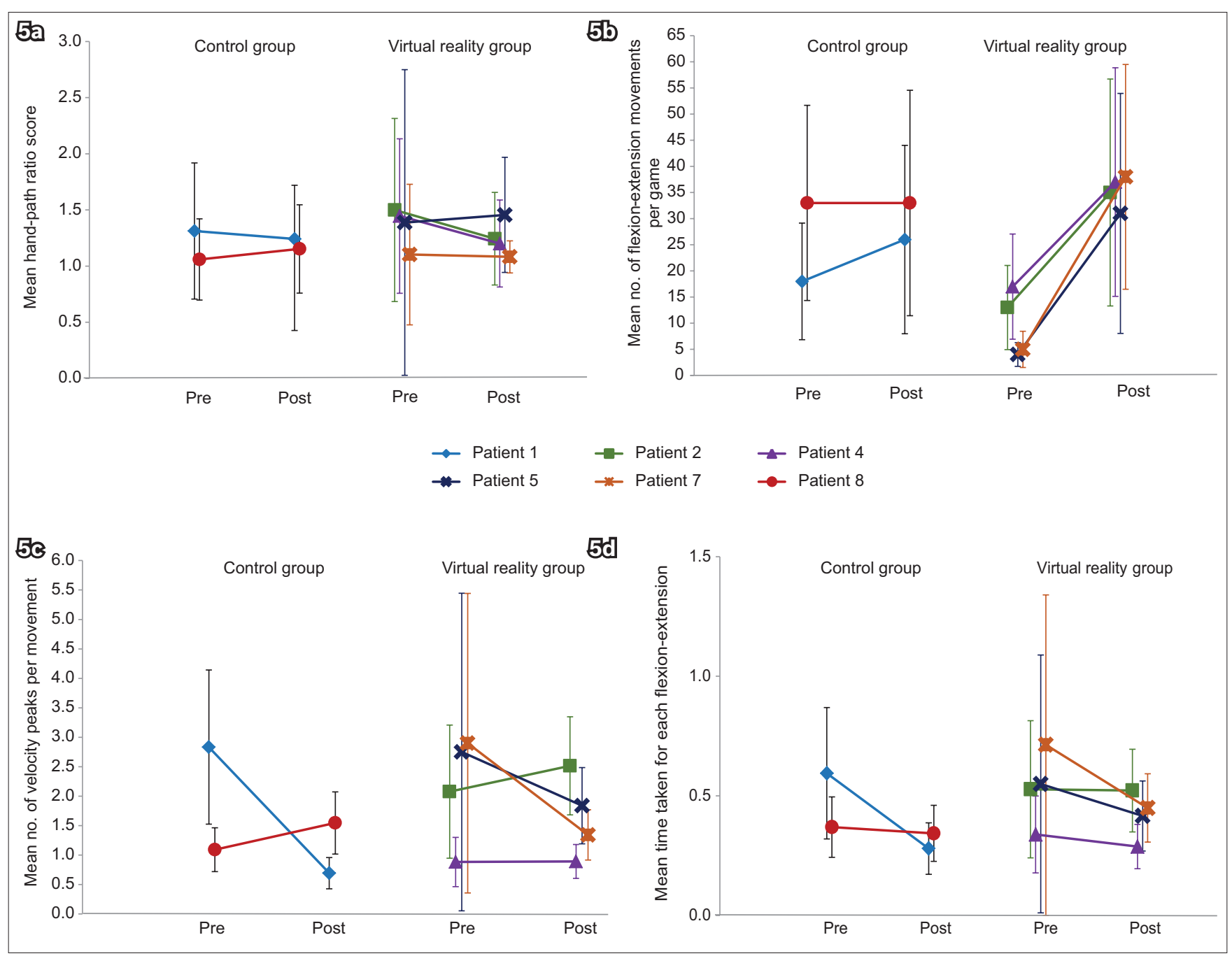

Fig. 5 Line graphs comparing pre- and post-trial assessment scores of the control and virtual reality group patients show changes in the kinematics of upper limb movement, as measured by mean (a) hand-path ratio scores, (b) number of flexion-extension movements per game, (c) number of velocity peaks per movement, and (d) time taken for each movement, in patients who underwent combination therapy.

found VR therapy effective and engaging. Some requested for it to be part of routine therapy. When they were shown a visual trend of their daily improvement in kinematic measures, patients responded that they could see how well they were performing each day. Selected responses from the questionnaire are shown in Fig. 6.

\section{DISCUSSION}

In the present study, we observed an improvement in clinical measures of arm function, as assessed by the FM-UE and ARAT, in patients undergoing combination therapy consisting of levodopa and VR-based intervention, despite having a short intervention period of two weeks. By incorporating an IMU-based bedside VR 


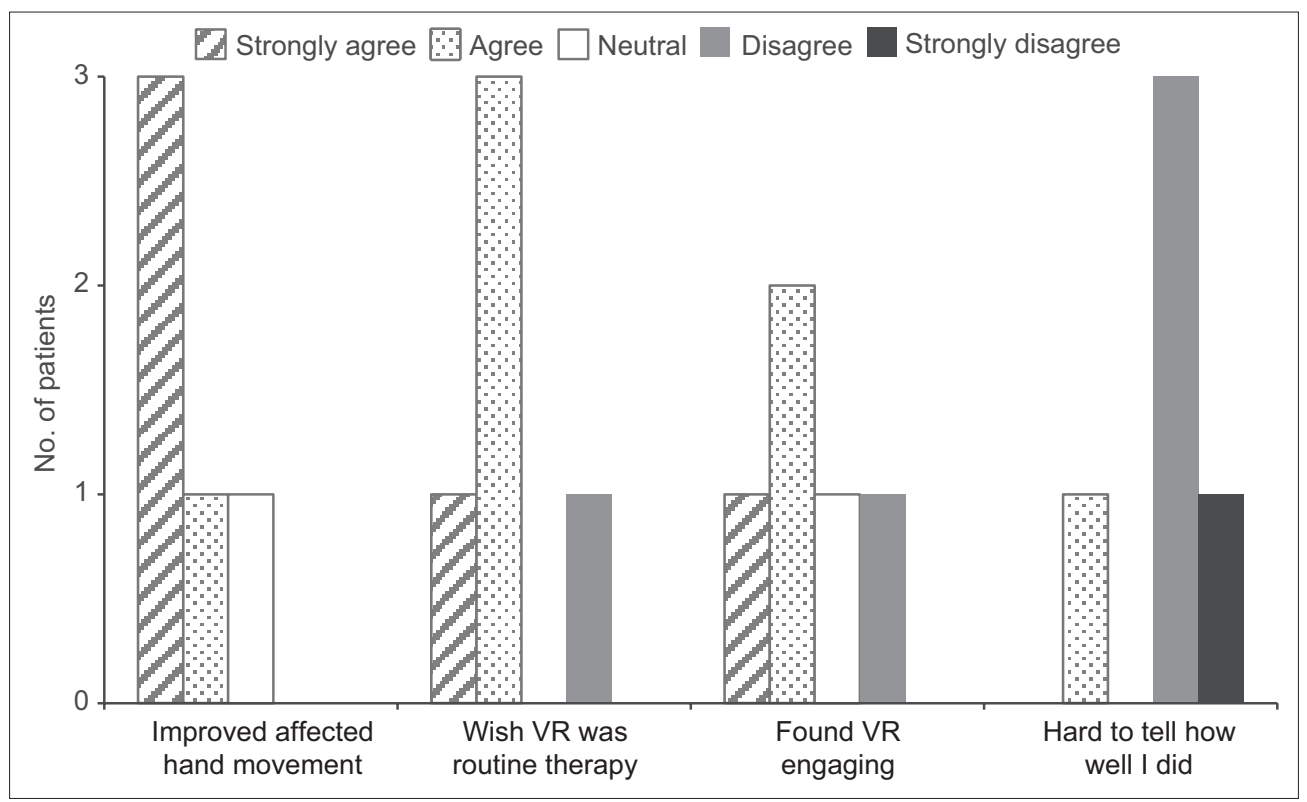

Fig. 6 Chart shows patients' responses to some questions about the virtual reality (VR)-based game in the questionnaire.

rehabilitation system, we were able to objectively assess the patients' kinematic measures in each session. Their HPR, VPs, time taken and flexions were found to improve, converging toward the scores of their nonparetic arms by the end of the two-week intervention.

We compared our results with three other studies that used VR-based rehabilitation in acute stroke patients. Kiper et al obtained improvements of $17.6 \%$ and $17.9 \%$ in FM-UE and Functional Independence Measure scores, respectively, ${ }^{(25)}$ while Saposnik et al obtained a $20.1 \%$ clinical improvement on the Box and Block Test ${ }^{(26)}$ and da Silva Cameirão et al attained a remarkable $46.0 \%$ improvement in FM-UE scores. ${ }^{(27)}$ None of these studies used neuromodulatory medication. Our intervention study group achieved a notable $45.2 \%$ improvement in FM-UE scores and 98.7\% improvement in ARAT scores.

Statistical analysis on the strength of the relationship between the clinical data and the kinematic data was not performed in view of the inadequate number of patients in our study. However, the improvements seen during clinical testing were likely to be matched by quantitative improvements in movement quality, as measured by kinematics, given that the FM-UE and ARAT scores improved, and the values for HPR, VP and time taken for each flexion-extension movement decreased, indicating an enhanced quality of movement.

Our findings have implications for the elucidation of neurobiological mechanisms that inform the optimal rehabilitation strategy in the acute post-stroke period. Early reports demonstrating the effectiveness of levodopa suggested that it may potentiate neuronal rewiring, ${ }^{(28)}$ but this effect could be highly reliant on the type of rehabilitative intervention. ${ }^{(29)}$ Since dopamine is a neurotransmitter that is associated with learning and memory of response-reward associations, ${ }^{(30)}$ we postulated that other factors that increase a patient's motivation to participate in rehabilitation may synergise with dopaminergic neuromodulation, leading to enhanced motor learning. Our simple VR gaming system served this function, as it was able to: (a) train a purposeful synergistic movement (bringing food to one's mouth) in a semi-immersive environment; (b) provide direct feedback on quantifiable daily improvement in kinematic parameters; and (c) enhance positive reinforcement through the use of stimulating audiovisual visuomotor feedback. Therefore, it can be hypothesised that although pharmacologic neuromodulation may set the stage for brain plasticity, the optimal rehabilitation protocol should incorporate factors such as visual, auditory and tactile feedback in order to be effective. As we have presented, a VR environment can have a simple setup and high ease of use. Hence, it would be useful for future studies to investigate the integration of pharmacotherapy and VR-based therapy in stroke rehabilitation and dissect the exact contributions made by each modality. Although photosensitive epilepsy has been known to be triggered by visual stimulation from video games, seizures that occur after stroke are more closely associated with factors such as the severity and location of the stroke, and with haemorrhagic strokes. ${ }^{(31,32)}$ In addition, there is no significant evidence that stroke may lower the threshold for seizures. Nevertheless, when using VR-based therapy with stroke patients, it may be prudent to be mindful of the risk of seizures during the course of visual stimulation.

The strong correlations between most kinematic measures obtained in this study and the clinical measurements are important contributions in the search for a more objective, quantitative way of tracking rehabilitation outcomes. Kinematic measures have been shown to correlate well with clinical measures of upper extremity function in previous studies. ${ }^{(10,14,16)}$ Individual kinematic parameters may represent certain aspects of neurological function, such as motor function or coordination, but our results show that kinematic measures correlate more strongly with total FM-UE scores and thus can represent overall function of the trained upper extremity.

To the best of our knowledge, the use of kinematic outcome measures has not yet been integrated as a form of regular feedback on patients' daily improvements, which are often overlooked 
or indiscernible. One study developed a customised game in which difficulty levels could be adjusted autonomously based on speed, range of movement and latency to movement onset, but feedback on these parameters was not given to patients to show their improvements. ${ }^{(27)}$ Another study used kinematic measures, in addition to clinical scales, as baseline and post-intervention outcome measures for the effectiveness of VR rehabilitation in chronic stroke patients, but there was no information on whether this data was communicated to patients. ${ }^{(33)}$ By comparison, our system allowed for session-by-session graphical feedback to patients on their performance improvements during the game sessions.

With the increasing availability and development of wearable sensors that capture movement, there is compelling potential in the use of kinematic measures as outcome measures for stroke rehabilitation. Not only are these measures more sensitive and objective, but their ability to quantitatively track patients' progress while they undergo rehabilitation also removes the need for therapists to perform additional evaluation sessions. Based on the preliminary results provided by this study, we postulate that kinematic outcome measures may benefit all the key players in rehabilitation, namely patients, clinicians, therapists and researchers: patients remain motivated by seeing their daily improvements, clinicians and therapists obtain information necessary to intervene in a timelier manner, and researchers acquire a more objective and quantitative way of tracking outcomes across different sites.

There were several limitations to our study. The clinical numbers were too small to arrive at firm conclusions about the clinical significance and efficacy of VR-based therapy combined with pharmacotherapy in acute stroke rehabilitation. However, there was a trend toward improvement in the VR group that should be verified in future studies. According to the patient feedback, one probable reason behind the poor recruitment rate was that many patients were uncomfortable with taking an additional pharmacological agent for the purpose of the trial. Also, the initial functional levels of the control group were high, due to the small patient numbers and its subsequent dropouts, leading to a ceiling effect on the improvement that this group made.

Ideally, we would have had a third control arm of patients who were not given any additional pharmacological or VR therapy, to account for spontaneous recovery from stroke, but this was not undertaken because of manpower and time constraints. Instead, it was postulated that the trial control group would improve at least as much as patients who were undergoing a conventional stroke rehabilitation programme. Therefore, we are planning for a larger follow-up study with such a third arm to control for spontaneous recovery. In addition, we were unable to fully control the type and intensity of the additional occupational therapy that control group patients received, as the direction of therapy is often dependent on the interactions between the patient and therapist. Nonetheless, we attempted to mimic the VR therapeutic intervention by instructing the occupational therapist to focus on arm flexion-extension exercises as far as possible.

A specific limitation concerning the IMUs would be gyroscope drift due to the inherent design of the small form-factor MEMS utilised in studies of this type. This is a challenging issue, as individual sensors have different drifting patterns and algorithm refinements require further development. Lastly, patients were only followed up for two weeks in this study, which was too short an evaluation period to determine if VR therapy resulted in sustained improvements, and only two out of the ten patients recruited returned for review at the third month after discharge.

In conclusion, this pilot study demonstrated that it is feasible to combine VR-based therapy and pharmacotherapy for acute stroke rehabilitation and to gather objective data for analysis. Daily kinematic measurements were found to be a useful additional tool for tracking upper limb performance. Future studies with larger numbers of patients and longer follow-up are needed to evaluate if the combination of VR-based therapy and pharmacotherapy in the acute stages after stroke significantly improves upper limb recovery, both in terms of rate and degree of eventual recovery, and whether this effect is sustainable and clinically significant in the long term.

\section{ACKNOWLEDGEMENTS}

This research was supported by the National Research Foundation, Prime Minister's Office, Singapore, under its IDM Futures Funding Initiative and administered by the Interactive and Digital Media Programme Office. This research is also part of an ongoing research initiative funded by the Singapore General Hospital Department of Medicine's Pitch For Funds Grant.

\section{REFERENCES}

1. Sun $\mathrm{Y}$, Lee $\mathrm{SH}$, Heng $\mathrm{BH}$, Chin VS. 5-year survival and rehospitalization due to stroke recurrence among patients with hemorrhagic or ischemic strokes in Singapore. BMC Neurol 2013; 13:133.

2. Go AS, Mozaffarian D, Roger VL, et al; American Heart Association Statistics Committee and Stroke Statistics Subcommittee. Heart disease and stroke statistics--2014 update: a report from the American Heart Association. Circulation 2014; 129:e28-292.

3. Nichols-Larsen DS, Clark PC, Zeringue A, Greenspan A, Blanton S. Factors influencing stroke survivors' quality of life during subacute recovery. Stroke 2005; 36:1480-4

4. Horn SD, Dejong G, Smout RJ, et al. Stroke rehabilitation patients, practice, and outcomes: is earlier and more aggressive therapy better? Arch Phys Med Rehabil 2005; 86(12 Suppl 2):S101-S114.

5. Krakauer JW, Carmichael ST, Corbett D, Wittenberg GF. Getting neurorehabilitation right: what can be learned from animal models? Neurorehabil Neural Repair 2012; 26:923-31.

6. Stinear C, Ackerley S, Byblow W. Rehabilitation is initiated early after stroke, but most motor rehabilitation trials are not: a systematic review. Stroke 2013; 44:2039-45.

7. Molina K, Ricci N, de Moraes S, Perracini M. Virtual reality using games for improving physical functioning in older adults: a systematic review. J Neuroeng Rehabil 2014; 11:156.

8. Dobkin BH. Strategies for stroke rehabilitation. Lancet Neurol 2004; 3:528-36.

9. Engelter ST. Safety in pharmacological enhancement of stroke rehabilitation. Eur J Phys Rehabil Med 2013; 49:261-7.

10. Laver K, George S, Thomas S, Deutsch JE, Crotty M. Cochrane review: virtual reality for stroke rehabilitation. Eur J Phys Rehabil Med 2012; 48:523-30.

11. Fluet GG, Deutsch JE. Virtual Reality for Sensorimotor Rehabilitation Post-Stroke: The Promise and Current State of the Field. Curr Phys Med Rehabil Rep 2013; 1:9-20.

12. Fluet GG, Merians AS, Qiu Q, Davidow A, Adamovich SV. Comparing integrated training of the hand and arm with isolated training of the same effectors in persons with stroke using haptically rendered virtual environments, a randomized clinical trial. J Neuroeng Rehabil 2014; 11:126.

13. Saposnik G, Mamdani M, Bayley M, et al; EVREST Study Group for the Stroke Outcome Research Canada Working Group. Effectiveness of Virtual Reality 
Exercises in STroke Rehabilitation (EVREST): rationale, design, and protocol of a pilot randomized clinical trial assessing the Wii gaming system. Int J Stroke 2010; 5:47-51.

14. Gebruers N, Truijen S, Engelborghs S, De Deyn PP. Prediction of upper limb recovery, general disability, and rehabilitation status by activity measurements assessed by accelerometers or the Fugl-Meyer score in acute stroke. Am J Phys Med Rehabil 2014; 93:245-52.

15. Yin CW, Sien NY, Ying LA, Chung SF, Tan May Leng D. Virtual reality for upper extremity rehabilitation in early stroke: a pilot randomized controlled trial. Clin Rehabil 2014; 28:1107-14.

16. Bosecker C, Dipietro L, Volpe B, Krebs HI. Kinematic robot-based evaluation scales and clinical counterparts to measure upper limb motor performance in patients with chronic stroke. Neurorehabil Neural Repair 2010; 24:62-9.

17. van Dokkum L, Hauret I, Mottet D, et al. The contribution of kinematics in the assessment of upper limb motor recovery early after stroke. Neurorehabil Neural Repair 2014; 28:4-12.

18. Cattaneo L, Rizzolatti G. The mirror neuron system. Arch Neurol 2009; 66:557-60.

19. Rohrer B, Fasoli S, Krebs HI, et al. Movement smoothness changes during stroke recovery. J Neurosci 2002; 22:8297-304.

20. Broeren J, Sunnerhagen KS, Rydmark M. A kinematic analysis of a haptic handheld stylus in a virtual environment: a study in healthy subjects. J Neuroeng Rehabil 2007; 4:13.

21. Thielman GT, Dean CM, Gentile AM. Rehabilitation of reaching after stroke: task-related training versus progressive resistive exercise. Arch Phys Med Rehabil 2004; 85:1613-8.

22. Holden MK, Dyar TA, Dayan-Cimadoro L. Telerehabilitation using a virtual environment improves upper extremity function in patients with stroke. IEEE Trans Neural Syst Rehabil Eng 2007; 15:36-42.

23. Page SJ, Fulk GD, Boyne P. Clinically important differences for the upper- extremity Fugl-Meyer Scale in people with minimal to moderate impairment due to chronic stroke. Phys Ther 2012; 92:791-8.

24. Lang CE, Edwards DF, Birkenmeier RL, Dromerick AW. Estimating minimal clinically important differences of upper-extremity measures early after stroke. Arch Phys Med Rehabil 2008; 89:1693-700.

25. Kiper P, Piron L, Turolla A, Stozek J, Tonin P. The effectiveness of reinforced feedback in virtual environment in the first 12 months after stroke. Neurol Nuerochir Pol 2011; 45:436-44.

26. Saposnik G, Teasell R, Mamdani M, et al; Stroke Outcome Research Canada (SORCan) Working Group. Effectiveness of virtual reality using Wii gaming technology in stroke rehabilitation: a pilot randomized clinical trial and proof of principle. Stroke 2010; 41:1477-84.

27. da Silva Cameirão M, Bermúdez I Badia S, Duarte E, Verschure PF. Virtual reality based rehabilitation speeds up functional recovery of the upper extremities after stroke: a randomized controlled pilot study in the acute phase of stroke using the rehabilitation gaming system. Restor Neurol Neurosci 2011; 29:287-98.

28. Scheidtmann K, Fries W, Müller F, Koenig E. Effect of levodopa in combination with physiotherapy on functional motor recovery after stroke: a prospective, randomised, double-blind study. Lancet 2001; 358:787-90.

29. Sonde L, Lökk J. Effects of amphetamine and/or L-dopa and physiotherapy after stroke - a blinded randomized study. Acta Neurol Scand 2007; 115:55-9.

30. Wise RA. Dopamine, learning and motivation. Nat Rev Neurosci 2004; 5:483-94.

31. Hughes JR. The photoparoxysmal response: the probable cause of attacks during video games. Clin EEG Neurosci 2008; 39:1-7.

32. Arboix A, García-Eroles L, Massons JB, Oliveres M, Comes E. Predictive factors of early seizures after acute cerebrovascular disease. Stroke 1997; 28:1590-4.

33. Piron L, Turolla A, Agostini M, et al. Exercises for paretic upper limb after stroke: a combined virtual-reality and telemedicine approach. J Rehabil Med 2009; 41:1016-102. 